\title{
Digitalization in Clinical Biochemistry: Need to Prepare for Future
}

Diagnostic laboratories form a crucial part of modern medicine, providing vital information for the prevention, treatment and management of a disease. The digital innovation in healthcare is also growing at an increasingly fast pace across specialties, and the laboratory is no exception. The role of the medical laboratories, which, classically, is to provide information to the clinician for evaluation and diagnosis, will change as laboratories may play a more active role in the future as a "nerve center of diagnostics".

Role of information technology in laboratories has largely been in the form of the lab information system (LIS), which is commonly used to manage quality control, patient test reports and inventory. However, this will expand as laboratories become an essential player in digital health, defined by the US Food and Drug Administration (FDA) as a field encompassing wearable devices, mobile health (mHealth), telemedicine, personalized medicine, electronic health records (EHR), and healthcare information technology (IT).

The Government of India has started the ambitious National Digital Health Mission (NDHM), which aims to develop the backbone necessary to support the integrated digital health infrastructure of the country for universal health coverage in an efficient, accessible, inclusive, affordable, timely and safe manner. As patients will be able to securely store and access their medical records (such as prescriptions, diagnostic reports and discharge summaries), and share them with healthcare providers, laboratories will have to adopt these uniform standards, so that their reports can be integrated with the patient's EHR. Laboratories will also have better access to a patient's medical history from this digital database, which will help labs to interpret the test results better. People are already ordering test packages themselves as part of routine health check-ups, without any referral from a physician. For such patients directly coming to labs, appropriate advice and clinician referrals will also be expected. NDHM will also help digitize the claims process and enable faster reimbursement, enhancing the overall ease of providing services amongst the healthcare providers.

Already, wearable smart devices with capability to monitor various parameters, such as pulse rate and oxygen saturation levels are available. Wearable devices for non-invasive continuous monitoring of glucose through the skin are also available, which allow patients to see glucose levels, trends and alerts on their mobile device. Smart devices which track physical activity and food intake will also contribute valuable health data. As the range of parameters monitored by such devices expands, the data generated by these will have to be considered by the laboratories for overall patient management.

Artificial intelligence (Al) is also making inroads in healthcare, and an increasing number of Al-based algorithms have been approved by the FDA, mainly in the areas of cardiology and radiology. Machine learning, which can be used to create medical Al, is defined as "the study of how computer algorithms can 'learn' complex relationships or patterns from empirical data and produce (mathematical) models linking a large number of covariates to some target variable of interest". Models have been developed and tested to predict the survival of patients with heart failure from serum creatinine and ejection fraction. Other such models include predicting the risk of polyneuropathy in diabetic patients and prediction of SARS-CoV-2 infection using routine laboratory blood tests.

Technological innovation in healthcare with information integration offers potential opportunities for laboratories to evolve toward a higher level of visibility, as the center of diagnostic information rather than being the hidden support service. Integration of patient data from multiple sources for test result reporting will need upgradation of infrastructure as well as skill. Laboratories need to prepare for this transformation, by planning their IT resources and training of the personnel, so that they are able to rapidly adapt to these requirements and assert their important role in healthcare management.

Dr Rajeev Goyal Associate Professor Department of Biochemistry Lady Hardinge Medical College, New Delhi, India 